\title{
Revisiting the convergence of successive approximations in the problem of disturbed body motion
}

\author{
Vera Petelina ${ }^{1 *}$ \\ ${ }^{1}$ Moscow state university of civil engineering, Yaroslavskoye shosse, 26, Moscow, Russia, 129337
}

\begin{abstract}
This work discusses the problem of the convergence of Picard's successive approximations, solving the differential equation system of disturbed body motion through assembling a majorizing linear differential equation. The problem is to determine in which region of variables used these successive approximations will converge and to estimate the error, if a finite number of approximations is used.
\end{abstract}

\section{Introduction}

One of the most important mechanics problems is the approximation of orthogonal coordinates, body velocity components and time by algebraic polynomials of the lowest degree in relation to an auxiliary variable with a prescribed accuracy degree. Sundman realized the Poincare's idea of series generation by degrees of an independent variable convergent for any real time value, but the Sundman series converge very slowly and therefore have not practical application potential. Another way for solution of the threebody problem was specified by G.A. Merman in [1]: in his solution, orthogonal coordinates and time are represented as Mittag-Leffler series, members of which are polynomials in an independent regularizing variable. These polynomial series converge faster than the Sundman series, since the convergence region is broader. New methods for determination of disturbances in orthogonal coordinates preserve standard features of conventional methods. For disturbance calculation a small parameter method is used; it enables asymptotic solution expansion and applies the Picard method of motion equations integration. This method leads to a convergent process of successive approximations, providing a solution of the disturbed motion differential equation system. The solution error depends on the accuracy of the initial approximation of the disturbing function.

The work [3] provides the idea of orthogonal coordinates, regularized velocity and time components for disturbed body motion in the second approximation as polynomials by degrees of a certain regularizing variable. The same can be performed to find the third, the fourth and higher approximations, since analytical features of these functions are known. Thus, it provides an iterative process for finding a solution of the disturbed body motion differential equation system.

\footnotetext{
* Corresponding author: verapetelina51@gmail.com
} 


\section{Mathematical model}

Let us prove the convergence of successive approximations and determined conditions, when successive approximations converge to an exact solution. Let us consider a differential equation system of the following type:

$$
\frac{d C_{i}}{d u}=\varepsilon\left[f_{i 0}(u)+f_{i 1}\left(u, C_{1}, C_{2}, \ldots, C_{7}\right)\right](i=1,2, \ldots, 7)
$$

where: $\varepsilon$ - a small parameter,

$u$ - an independent variable, where $-1 \leq u \leq 1$

$C_{i},(i=1,2, \ldots, 7)$ - in the problem under review these are variations of osculating initial values of orthogonal coordinates $\xi, \eta, \zeta$, regularized velocity components $\xi^{\prime}, \eta^{\prime}, \zeta^{\prime}$, time point $\tau$.

$f_{i 0}(u)$ - right-hand members of the differential equation system [2, formulas (30)-(33)] at:

$$
\begin{gathered}
\xi=\xi_{0} ; \eta=\eta_{0} ; \zeta=\zeta_{0} ; \xi^{\prime}=\xi_{0}^{\prime} ; \eta^{\prime}=\eta_{0}^{\prime} ; \zeta^{\prime}=\zeta_{0}^{\prime} ; \tau=\tau_{0} . \\
f_{i 1}\left(u, C_{1}, C_{2}, \ldots, C_{7}\right)=f_{i}\left(u, C_{1}+\xi_{0}, C_{2}+\eta_{0}, \ldots, C_{7}+\tau_{0}\right)-f_{i}\left(u, \xi_{0}, \eta_{0}, \ldots, \tau_{0}\right)
\end{gathered}
$$

$f(u, \xi, \eta, \ldots, \tau)$ - right-hand members of the differential equation system under review [2, formulas (30)-(33)]. The small parameter $\varepsilon$ is introduced for further representation of the solution of the differential equation system (1) as series by degrees $\varepsilon$.

It is possible to select such $C^{*}$ that in the hyperparallelepiped $D$ : $-1 \leq u \leq 1 ;\left|C_{i}\right| \leq C^{*}$, the right-hand member of the system (1) will be continuous in $u$ and will satisfy generalized Lipschitz conditions (4):

$$
\left|f_{i 1}\left(u, C_{1}^{*}, C_{2}^{*}, \ldots, C_{7}^{*}\right)-f_{i 1}\left(u, C_{1}^{* *}, C_{2}^{* *}, \ldots, C_{7}^{* *}\right)\right| \leq \sum_{j=1}^{7} L_{i j}(u) \cdot\left|C_{j}^{*}-C_{j}^{* *}\right|
$$

where: $L_{i j}(u)=\sup \left|\frac{\partial f_{i 1}}{\partial C_{j}}\right|$ in the hyperparallelepiped $D_{u}$, which is a cross-section of the hyperparallelepiped $D$ by the plane $u=u_{0}$.

Let us find a solution of the differential equation system (1) by the successive approximations method. Assuming $C_{i}=0,(i=1,2, \ldots, 7)$, in the first approximation it is:

$$
C_{i}^{(1)}(u)=\varepsilon \cdot \int_{-1}^{u} f_{i 0}(u) d u
$$

in the second approximation it is:

$$
C_{i}^{(2)}(u)=\varepsilon \cdot \int_{-1}^{u}\left(f_{i 0}(u)+f_{i 1}\left(u, C_{1}^{(1)}(u), \ldots, C_{7}^{(1)}(u)\right)\right) \cdot d u,
$$

the $\mathrm{k}$-th approximation it is:

$$
\begin{gathered}
C_{i}^{(k)}(u)=C_{i}^{(1)}(u)+\varepsilon \cdot \int_{-1}^{u} f_{i 1}\left(u, C_{1}^{(k-1)}(u), \ldots, C_{7}^{(k-1)}(u)\right) \cdot d u= \\
=\varepsilon \cdot \int_{-1}^{u}\left(f_{i 0}(u)+f_{i 1}\left(u, C_{1}^{(k-1)}(u), \ldots, C_{7}^{(k-1)}(u)\right)\right) \cdot d u
\end{gathered}
$$


Let us review the auxiliary functions $P(u)$ and $Q(u)$, for which:

$$
\left|f_{i 0}(u)\right| \leq P(u) ; \sup \left|\frac{\partial f_{i 1}}{\partial C_{j}}\right|=L_{i j}(u) \leq L(u) ;|Q(u)|=7 \cdot L(u)
$$

$(i, j=1,2, \ldots, 7)$

Let us generate a majorizing linear differential equation with variable coefficients:

$$
\frac{d C}{d u}=\varepsilon[P(u)+Q(u) \cdot C]
$$

which is reviewed within a range of $-1 \leq u \leq 1$.

Let us analyze the solution convergence for the equation (9), which is as follows:

$$
C(u)=e^{\varepsilon \cdot \int_{-1}^{u} Q(u) \cdot d u} \cdot \varepsilon \cdot \int_{-1}^{u} P(u) \cdot e^{-\varepsilon \cdot \int_{-1}^{u} Q(u) \cdot d u} \cdot d u
$$

where $C(-1)=0$. It can be proved that $C^{\prime}(u) \succ 0, C(u)$ is a steadily increasing function and is represented as an absolutely convergent series:

$$
C(u)=\sum_{k=1}^{\infty} \varepsilon^{k} \cdot \widetilde{C}^{k}(u),
$$

If:

$$
C(1)=e^{\varepsilon \cdot \int_{-1}^{l} Q(u) \cdot d u} \cdot \varepsilon \cdot \int_{-1}^{l} P(u) \cdot e^{-\varepsilon \cdot \int_{-1}^{u} Q(u) \cdot d u} \cdot d u \leq C^{*} .
$$

Let us find a solution for the equation (9) by the successive approximations method. After $l$-iterations we have:

$$
C^{(l)}(u)=\sum_{1}^{(l)} \varepsilon^{k} \cdot \widetilde{C}^{k}(u),
$$

where: $\varepsilon^{k} \cdot \widetilde{C}^{k}(u)=C^{(k)}(u)-C^{(k-1)}(u), \quad C^{(k)}(u)$ - solution of the majorizing equation (9) in $\mathrm{k}$-th approximation.

Let us now prove that if the condition (12) is satisfied, successive approximations, representing the solution of the system (1), converge to functions that represent the solution of the equation (9). Considering the conditions (4) and the formulas (7), we have:

$$
\begin{gathered}
\left|C_{i}^{(k)}-C_{i}^{(k-1)}\right| \leq \varepsilon \cdot \int_{-1}^{u}\left[f_{i 1}\left(u, C_{1}^{(k-1)}, C_{2}^{(k-1)}, \ldots, C_{7}^{(k-1)}\right)-f_{i 1}\left(u, C_{1}^{(k-2)}, C_{2}^{(k-2)}, \ldots, C_{7}^{(k-2)}\right)\right] \cdot d u \leq \\
\leq \varepsilon \sum_{j=1}^{7} \int_{-1}^{u} L_{i j}(u) \cdot\left|C_{j}^{(k-1)}-C_{j}^{(k-2)}\right| \cdot d u
\end{gathered}
$$

Supposing that the inequality:

$$
\left|C_{i}^{(m)}-C_{i}^{(m-1)}\right| \leq \varepsilon^{m} \cdot \widetilde{C}^{(m)}(u)
$$

is true for $(m=1,2, \ldots, k-1)$, we will know that is true for $(m=k)$. It follows from the inequality (14). Thus, if the condition (12) is satisfied, the inequality (15) is true with any $m$, i.e. successive approximations, representing the solution of the system (1), converge to functions, representing the solution of the majorizing equation (9). If the solution of the equation (9) exits the hyperparallelepiped at $-1<\alpha<1$, i.e. at $C(\alpha)=C^{*}$, the successive approximations $C_{i}^{(m)}(u)$ absolutely converged within $[-1 ; \alpha]$. If we estimate $C^{*}$, where 
$\left|C_{i}\right| \leq C^{*},(i=1,2, \ldots, 7)$, we can find a time interval, where the approximations under review converge.

\section{Assembling a majorizing linear differential equation}

Let us consider the simplest variant of the problem, when the right-hand equation member of the system [2 of the formulas (30) - (33)] and the functions $L_{i j}(u)$ are amplified in the hyperparallelepiped $D-1 \leq u \leq 1,\left|C_{i}\right| \leq C^{*}$ by their maximum values.

$$
\max P(u)=A, \quad \max Q(u)=B
$$

The usage of regularized differential equations [2 of the formulas (30) - (33)] will automatically remove singularities due to collision of the body with the Earth. The convergence of successive approximations for regularized equations depends on the error in selection of initial approximations of unknown boundary conditions to a much lesser degree.

Let us assume that $\varepsilon=1$, and the equation (9) will appear as follows:

$$
\frac{d C}{d u}=A+B \cdot C
$$

and its solution will be:

$$
C(u)=\frac{A}{B}\left(e^{B u}-1\right)
$$

Let us set the right-hand part (18) equal to the value $C^{*}$, and we will receive the following transcendental equation:

$$
\frac{A}{B}\left(e^{B u}-1\right)=C^{*}
$$

from which we can determine the value of the independent regularizing variable $u=u_{1}$, corresponding to $C(u)=C^{*}$.

$$
u_{1}=\frac{1}{B} \cdot \ln \left(\frac{B \cdot C^{*}}{A}+1\right)
$$

For estimation of the value $C^{*}$ it is necessary to estimate values $\mathrm{A}$ and $\mathrm{B}$. For estimation of the value $A$ it is necessary to evaluate the right-hand parts of the differential equation system for disturbed body motion, where the desired variables are osculating initial values of orthogonal coordinates $\xi, \eta, \zeta$, components of regularized velocity $\xi^{\prime}, \eta^{\prime}, \zeta^{\prime}$, regularized acceleration $\xi^{\prime \prime}, \eta^{\prime \prime}, \zeta^{\prime \prime}$, the time point $\tau$, the radius-vector $\rho$ and its derivatives $\rho^{\prime}$ and $\rho^{\prime \prime}$ by the independent regularizing variable $u$ considering the assumptions made. For this purpose, the osculating initial values of orthogonal coordinates, components of regularized velocity, regularized acceleration and time of geocentric motion of the body are supplemented with members dependent of the parameter $C^{*}$, assumed with their scaling factors, i.e. the members $C_{i}=\gamma_{i} C^{*}(i=1,2, \ldots, 7)$, where the scaling factors $\gamma_{1}, \gamma_{2}, \gamma_{3}$ are dimensionless, $\gamma_{4}, \gamma_{5}, \gamma_{6}$ are dimension factors $(\mathrm{km} / \mathrm{s}), \gamma_{7}$ is a dimension factor $(\mathrm{s} / \mathrm{km})$. For each function in the right-hand parts of the differential equation system [2 of the formulas (30) - (33)] the right and left boundaries were estimated. They depend on the value $C^{*}$, and to find the left boundary the minimum value was sought after, and the components $\gamma_{i} C^{*}, \quad(i=1,2, \ldots, 7)$ were assumed with the minus sign; to find the right boundary the maximum value was sought after. The maximum-modulus estimate was 
selected as the desired estimate. Thus, rough function estimates were produced, using mainly geometrical considerations. More precise estimates of these functions are built considering behavior of functions $x, y, z, x^{\prime}, y^{\prime}, z^{\prime}, \xi^{\prime \prime}, \eta^{\prime \prime}, \zeta^{\prime \prime}, \sigma$ and their derivatives with respect to the variable $\rho$. Note that the inequality (12) includes only integral characteristics of the functions $P(u) Q(u)$.

This ensures a more precise generation of the majorizing equation (9), and the behavior of its solution does not differ much from the behavior of solution of the initial equation (1).

\section{An example of successive approximation convergence domain evaluation}

To illustrate the developed research methodology for the convergence of successive approximations, calculations were carried out with specified initial conditions, parabolic motion conditions of the body, considering assumptions made, and this resulted in the numerical estimate of the convergence domain. Several variants were calculated with different values of the variable $u$; the dependence of the variable $u$ on $C^{*}$ was established, and it was proved that this dependence has the extremum.

Dependence of the parameter $\mathrm{C}^{*}$ on the quantity of the scale factors $\gamma_{i}(\mathrm{i}=1,2,3,4,5,7)$ was analyzed. In the case of degeneracy the dependence of the quantity $\mathrm{C}^{*}$ on the variable $u$ has no extremum with $\gamma_{i}=0(\mathrm{i}=1,2,3,4,5,7)$, since the quantities $\mathrm{A}$ and $\mathrm{B}$ do not depend on the $\mathrm{C}^{*}$. When increasing the factors $\gamma_{i}$, this dependence has an extremum, which indicates an extension of the convergence domain. The quantity $\mathrm{C}^{*}$ is limited, so it can be assumed that the scale factors fall within a certain range of variation.

When giving the value of the parameter $\mathrm{C}^{*}$, it was defined more precisely using the developed technique. For the given initial conditions, we found the extremal value of the quantity $\mathrm{C}^{*}=4,987$ and the corresponding value of the independent variable $u_{1}=0.24$. Thus, if the mismatches in the rectangular coordinates of a body perturbed motion at the same instants of time at different stages of approximation do not exceed the quantity $C^{*}=4,987$ the successive approximations which represent the solution of the perturbed motion differential equations system converge absolutely.

Figure 1 shows the dependence of the parameter $\mathrm{C}^{*}$ on the standardized regularizing variable $u$ (the dotted line shows the dependence in the case of degeneracy with $\gamma_{i}=0$, the solid line shows the same with $\gamma_{1}=\gamma_{2}=0.08 \quad \gamma_{4}=\gamma_{5}=0.001$. 


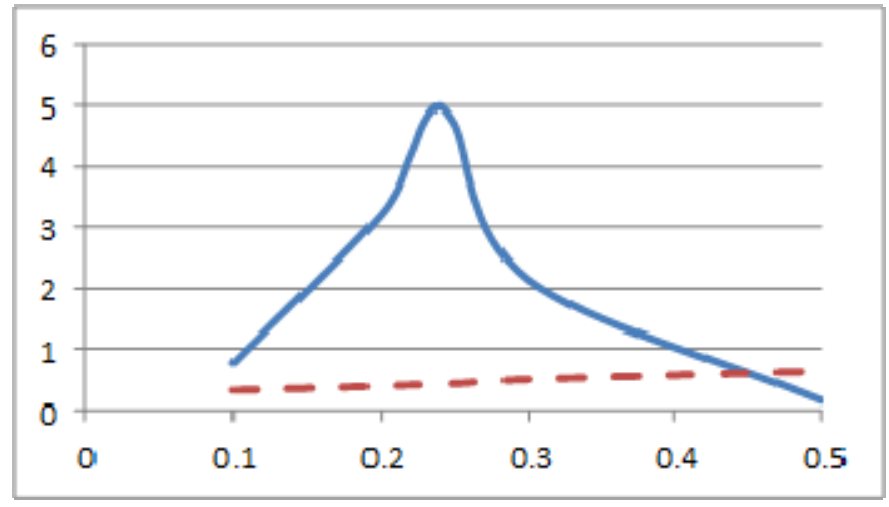

Fig. 1. The dependence of the parameter « $\mathrm{C}^{*} »$ on the normalized regularizing variable $« u$ »

\section{Conclusions}

A research method for the convergence of Picard successive approximations, representing a solution of the differential equation system for disturbed body motion, was developed. A method for finding majorizing function estimates, required for determination of the of successive approximations convergence, was developed. These estimates were determined and analytical formulas for determination of the convergence domain boundaries were produced.

The developed technique for study of the Picard's successive approximations convergence which represents the solution of the body perturbed motion differential equations system can be used in underground construction.

\section{References}

1. G.A. Merman. On representation of the general solution of the three-body problem using convergent series. Bulletin of the USSR AS Theoretical Astronomy Institute, 6, 10, 713-731 (1958)

2. V.D. Petelina. Disturbances of the second and higher orders for geocentric body motion. In the compilation "Universe study problems. Development of astronomy research methods", the USSR AS Theoretical Astronomy Institute, L. 8, 247-258. (1979)

3. M.S. Yarov-Yarovoy. Analytic theory of body motion toward the Moon. Materials of the P.K. Sternberg's State Astronomy Institute, 36, 28-160 ( 1967) 